specificity and the sensitivity to quantify biological processes in vivo. However, PET tracer uptake does not usually provide sufficiently detailed anatomical structure to accurately allocate receptor activity to precise tissue regions. This may be overcome by simultaneous imaging with magnetic resonance imaging (MRI). The aim of the study was to evaluate the combined PET/MRI scanner for the investigation of biological processes in rodents. $\mathrm{N}$-(5-fluoro-2-phenoxyphenyl)-N-(2-[18F]-fluoroet hyloxy5 -methoxybenzyl)acetamide ([18F]FEDAA1106) binds the translocator protein (TSPO) which is up-regulated in activated macrophages and may quantify vascular inflammatory pathologies. Dynamic in vivo imaging was carried out using a modified Focus F120 microPET incorporated into a bespoke 1 Tesla MR magnet (1). The pharmacokinetic profile of [18F]-FEDAA was characterised in mice. Simultaneously acquired PET and MRI reconstructed images were aligned together and the combined images revealed rapid uptake of [18F]-FEDAA into the heart, liver, lungs, kidneys and brain. Time activity curves were constructed using regions of interest delineated by the MR images and showed the expected pharmacokinetic profile. Thus, the results show that the fused anatomical-functional image not only provides anatomical context to the PET data, but can also allow improved quantification by more accurately defining the region of radioactive emission.

(1) Hawkes RC, et al. Technol Cancer Res Treat. 2010; 9(1):53-60.

\section{RAS-ASSOCIATION DOMAIN FAMILY 1 ISOFORM A (RASSF1A) IS A NOVEL REGULATOR OF TNF-ALPHA SIGNALLING IN CARDIOMYOCYTES}

Presenter: Tamer Mohamed

doi:10.1136/heartjnl-2012-302951.008

TMA Mohamed, M Zi, A Maqsood, S Prehar, L Neyses, D Oceandy. Cardiovascular Medicine Research Group, School of Biomedicine, University of Manchester

Tumour necrosis factor-alpha; (TNF-alpha) plays key roles in the pathogenesis of heart failure. Cardiomyocytes express the TNFalpha; receptor (TNFR), however, the mechanism of TNF-alpha; signal transmission in cardiomyocytes is not completely understood. Recent studies showed that in cancer cells TNFR is regulated by Ras-association domain family 1 isoformA (RASSF1A). Therefore, we investigated whether RASSF1A modulates TNFalpha; signalling in cardiomyocytes. We used RASSF1A knockout (KO) mice and wild type (WT) controls and stimulated them with TNF-alpha; $(10 \mu \mathrm{g} / \mathrm{kg}$ i.v.). In WT mice acute treatment with low dose of TNF-alpha increased cardiac contractility and intracellular calcium transient amplitude, which is consistent with previously published data (Circulation 2004; 109:406-411). However, KO mice showed a blunted contractile response following acute TNFalpha treatment as indicated by the change in end systolic elastance (in vivo) and intracellular calcium transient amplitude (isolated adult cardiomyocytes). We also found that RASSF1A formed a molecular complex with TNF-alpha; receptor in cardiomyocytes and this interaction was essential in the recruitment of TRADD and TRAF2, the major downstream effectors of TNFalpha; signalling. By mapping the interaction domain we found that the C-terminal region of RASSF1A was responsible for the formation of TNF-alpha; receptor complex. Furthermore, using an adenoviral-mediate $d$ shRNA construct we found that cardiomyocytes lacking RASSF1A exhibited reduced activation of NFkB, a downstream target of TNF-alpha. Overall, our data indicate an essential role of RASSF1A in regulating TNF-alpha; signalling in cardiomyocytes, with RASSF1A being key in the formation of TNF receptor complex and in the signal transmission to the downstream targets.

\section{BIOMARKERS FOR DETECTION OF ACTIVATED MACROPHAGES IN ATHEROSCLEROSIS}

doi:10.1136/heartjnl-2012-302951.009

Presenter: Joseph Bird

'JLE Bird, 'L Burzynski, ${ }^{2}$ M Manavaki, ${ }^{3} \mathrm{JHF}$ Rudd, , $\mathrm{E}$ A Warburton, ${ }^{1} \mathrm{AP}$ Davenport. ${ }^{1}$ Clinical Pharmacology Unit; ' ${ }^{2}$ epartment of Medicine; ${ }^{3}$ Division of Cardiovascular Medicine; ${ }^{4}$ Department of Clinical Neurosciences, University of Cambridge, Addenbrooke's Hospital Cambridge CB2 200, UK

Atherosclerosis is the pathogenic mechanism underlying the majority of strokes, heart attacks and peripheral vascular disease. These are the principal causes of morbidity and mortality in the developed nations. The atherosclerotic lesion (or plaque) is characterised by a build up of lipid and inflammatory cells in the artery wall. Arterial stenosis is the current clinical predictor for endarterectomy, although macrophage burden in the lesion correlates strongly with likelihood of rupture and could be a better proxy of plaque vulnerability. Activated macrophages have up-regulated expression of translocator protein (TSPO), which is readily quantified by the positron emission tomography (PET)-based ligands (1) such as [11C](R)-1-(2-chlorophenyl)-N-methyl-N-(1-methylpropyl)-3isoquinoline-carboxamide ([11C](R)-PK11195). Studies examining TSPO plaque biodistribution frequently use CD68, a pan-phenotype marker, for identification of macrophage presence. However, this biomarker demonstrates some inconsistencies in these analyses (1). We hypothesise that more specific macrophage phenotype markers may perform better as correlates for TSPO PET-ligand binding. In this study, we examined the expression of phenotyperestricted macrophage markers in endarterectomy tissue, and correlated these with the distribution of $[3 \mathrm{H}](\mathrm{R})-\mathrm{PK} 11195$ using autoradiographic, immunohistochemical and immunofluorescence techniques. Autoradiographic and immunohistochemical images were digitised and co-registered to provide more accurate correlation of expression. We identified several markers of activated macrophage phenotype which have better correlation with $[3 \mathrm{H}]$ (R)-PK11195 binding patterns compared with CD68. These data indicate that TSPO PET ligands bind principally to activated macrophage phenotypes, and the phenotype markers have the potential to more accurately identify PET-ligand binding macrophages in ex vivo analysis.

1) Bird JL et al. Atherosclerosis, (2010), 210(2): 388-91.

\section{REGULATION OF MONOCYTE-ENDOTHELIAL CELL INTERACTIONS BY NEUTROPHIL-DERIVED MICROPARTICLES}

doi:10.1136/heartjnl-2012-302951.010

Presenter: Amanda Burnett

A Burnett, P Hellewell, V Ridger. Department of Cardiovascular Science, University of Sheffield, Sheffield, S10 2RX

Rationale Monocytes play a major role in atherosclerosis progression through migration into the arterial wall. Neutrophil involvement in atherosclerosis was previously only thought to be via enzymatic weakening of the fibrous cap. However, neutrophil depletion can delay atherogenesis and conversely increasing circulating neutrophil enhance plaque progression in mice. Lack of evidence for the presence of neutrophils in atherosclerotic plaques makes their role in disease progression less clear. We have found neutrophil-derived microparticles increase migration of neutrophils. Our hypothesis: neutrophil-derived microparticles increase endothelial cell-monocyte interactions and facilitate monocyte transendothelial migration.

Methodology Neutrophils were incubated with various agents to stimulate microparticle formation. Microparticles were characterised and quantified using a novel, standardised flow cytometry 